\title{
Uso da regressão linear para estimativa da relação entre a condutividade elétrica e a composição iônica da água de irrigação'
}

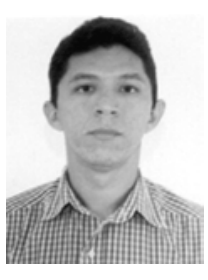

Giorgio M. Ribeiro² ${ }^{\text {, Celsemy E. Maia }{ }^{3} \text { \& José F. de Medeiros }}{ }^{3}$

1 Trabalho desenvolvido com apoio do convênio SINTEC/FUNPEC 008/2001
2 Escola Superior de Agricultura de Mossoró, CEP 59625-900. E-mail:giomendes@hotmail.com (Foto)
${ }^{3}$ Escola Superior de Agricultura de Mossoró, E-mail: celsemymaia@bol.com.br, jfmedeir@esam.br

Protocolo 78 - 3/6/2002 - Aprovado em 18/3/2004

Resumo: Vários estudos vêm sendo realizados ultimamente, com o propósito de se avaliar a qualidade de água de irrigação na região semi-árida do Nordeste brasileiro. Em alguns desses estudos, os autores têm ajustado diversas características químicas, como cálcio, magnésio, sódio e cloreto e soma de cátions, em função da condutividade elétrica (CE) através de equações empíricas; porém atenção deve ser dada às variações temporal e espacial dessas variáveis. Objetivouse, com o presente trabalho, avaliar a influência da fonte, da época e do tipo de solo sobre a condutividade elétrica, em função dos íons da água de irrigação, utilizando-se a regressão linear. Foi utilizado um banco de dados composto por 562 análises, oriundas de 55 propriedades rurais. As determinações químicas feitas nas amostras de águas, foram: $\mathrm{pH}, \mathrm{CE}, \mathrm{Ca}^{2+}, \mathrm{Mg}^{2+}, \mathrm{Na}^{+}, \mathrm{K}^{+}$, $\mathrm{Cl}^{-}, \mathrm{HCO}_{3}{ }^{-}, \mathrm{CO}_{3}{ }^{2-}$ e $\mathrm{SO}_{4}{ }^{2-}$. A partir de janeiro de 1988 realizaram-se amostragens nas propriedades, até 411 dias. $\mathrm{O}$ banco de dados foi dividido em 14 épocas de amostragem, três fontes (poço, rio e açude) e para 10 solos. Para se comparar as equações ajustadas, empregou-se o teste de identidade de modelo, cujos resultados mostraram que as equações lineares ajustadas com a condutividade elétrica em função dos teores de cálcio, magnésio, potássio, sódio, cloreto, bicarbonato, carbonato e sulfato variaram significativamente com a época de amostragem, a fonte de água e com o tipo de solo.

Palavras-chave: qualidade de água, salinidade, teste de identidade de modelo

\section{Use of linear regression to estimate the relationship between electrical conductivity and ionic composition of irrigation water}

\begin{abstract}
Several studies have been accomplished lately to evaluate irrigation water quality in the semi-arid region of the Northeast Brazil. In some of these studies, the authors have adjusted some chemical characteristics such as calcium, magnesium, sodium, chloride and sum of cations as a function of electrical conductivity (EC) through empirical equations, however attention should be given to temporal and spatial variations. In this paper, the influence of water source, time of sampling, and soil type in the linear regression determination of electrical conductivity as a function of the ions present in the irrigation water was evaluated. A 562-analysis database originated from 55 rural properties was utilized. The water samples were analyzed for $\mathrm{pH}, \mathrm{EC}$, $\mathrm{Ca}^{2+}, \mathrm{Mg}^{2+}, \mathrm{Na}^{+}, \mathrm{K}^{+}, \mathrm{Cl}^{-}, \mathrm{HCO}_{3}{ }^{-}, \mathrm{CO}_{3}{ }^{2-}$, and $\mathrm{SO}_{4}{ }^{2-}$. From January 1988 samplings in the properties were made during 411 days. The database was divided into 14 sampling times, three sources (well, river, and dam), and 10 soil types. For comparing the adjusted equations an identity model test was utilized. The adjusted linear equations with electrical conductivity as a function of the contents of calcium, magnesium, potassium, sodium, chloride, bicarbonate, carbonate, and sulfate varied significantly with time of sampling, water source, and soil type.
\end{abstract}

Key words: water quality, salinity, model identity test 


\section{INTRODUÇÃO}

A irrigação consiste em fornecer água às culturas quando não dispõem de quantidade suficiente para suprir suas necessidades; porém todo cuidado deve ser tomado quando se fizer uso da irrigação, pois alguns inconvenientes podem surgir, como a salinização, um dos principais problemas enfrentados devido, principalmente, à qualidade da água de irrigação e/ou ao manejo inadequado (Pizzarro, 1978; Servant, 1980).

A região semi-árida do Brasil, que corresponde a $52 \%$ do território do Nordeste brasileiro, apresenta características que permitem ainda mais o agravamento do quadro de salinização dos solos; em virtude da instabilidade climática, há escassez e intermitência de chuvas, associadas às altas taxas de evapotranspiração; no entanto, a prática de irrigação é indispensável na região em razão da ocorrência do déficit hídrico para as culturas, principalmente na época seca (Costa et al., 1982).

Dentre os problemas que a salinização pode causar ao solo, o aumento das forças de retenção da água caracterizase como fator importante, em razão de causar escassez de água às plantas e, conseqüentemente, redução e até a paralisação do crescimento, danos às folhas e tecidos das plantas, resultando em perdas no rendimento da maioria das culturas (Ayers \& Westcot, 1991). No que se refere à salinidade sobre os solos, Cruciani (1983) afirma que as propriedades físicas são afetadas pela concentração de sódio. Em decorrência do efeito dispersante do excesso desse íon no solo, a per-meabilidade fica comprometida, originando uma camada impermeabilizante, reduzindo drasticamente sua condutividade hidráulica. Essas alterações físico-químicas afetam o regime de umidade do solo, a aeração, nutrição e o desenvolvimento vegetativo e produtivo das plantas (Nunes Filho, 1984).

A salinidade dos solos consiste, atualmente, em um dos problemas mais preocupantes na agricultura moderna, devido a sua origem ser bastante variada, podendo ser de ordem marinha, lítica ou antropogênica. A salinização pode ocorrer em quase todas as áreas do planeta e uma das principais causas é o manejo, às vezes deficitário, das águas de irrigação, contribuindo para o aumento de perda da quantidade de solos (Farias, 1997).

A condutividade elétrica é a variável mais empregada para se avaliar o nível de salinidade, ou a concentração de sais solúveis na água de irrigação e no solo. Corresponde à medida da capacidade de uma água conduzir eletricidade e cresce proporcionalmente a medida em que a concentração de sais aumenta.

Vários estudos vêm sendo realizados ultimamente para se avaliar a qualidade de água de irrigação na região semi-árida do Nordeste brasileiro. Em grande parte desses estudos, alguns autores ajustaram os valores de cálcio, magnésio, sódio e cloreto e a soma de cátions, em função da condutividade elétrica (CE) através de equações empíricas. Martins (1993) ao estudar as relações empíricas a partir da CE, constatou que os coeficientes de correlação foram significativos $(p<0,001)$ para as diferentes características das águas estudadas e que, com exceção do $\mathrm{pH}, \mathrm{K}, \mathrm{CO}_{3}$ e $\mathrm{HCO}_{3}$, as demais características avaliadas apresentam pelo menos duas relações com coeficientes de correlação elevados ( $r>0,80)$. Medeiros (1992) encontrou dados semelhantes para as águas utilizadas na pequena irrigação dos Estados do Rio Grande do Norte, Paraíba e Ceará e com bons ajustes, utilizando a análise de regressão linear para algumas características químicas em função da condutividade elétrica. Resultado semelhante encontraram Nunes Filho et al. (2000) trabalhando com águas superficiais e subterrâneas do sertão de Pernambuco, onde concluíram que alguns íons podem ser estimados por meio da regressão linear, através da CE. Por outro lado, Maia et al. (1998) advertem para o fato de que, na determinação das concentrações de íons pela condutividade elétrica nas águas para irrigação, devem ser levados em consideração fatores determinantes na estimativa desses íons pela $\mathrm{CE}$, como a fonte e o local da amostragem.

Embora alguns modelos matemáticos e estatísticos propostos por diferentes autores expressem certo grau de confiabilidade bastante satisfatório, deve-se atentar para alguns aspectos envolvidos em questão, principalmente os citados por Maia et al. (1998). Sendo assim, é possível se obter resultados mais precisos para a avaliação da água de irrigação e a utilização de equações de ajuste de características físicoquímicas, a partir da condutividade elétrica.

O teste de identidade de modelo é útil para casos em que equações de regressão são ajustadas para diferentes locais e onde, em dois os mais locais, se podem utilizar a mesma equação de regressão sem prejuízo nas estimações. O teste pode ser utilizado, dentre outros métodos, para se analisar a possibilidade de uso de equações comuns a diferentes grupos de extratos e se baseia na diferença entre a soma de quadrados de parâmetros do modelo completo e a soma de quadrados de parâmetros do modelo reduzido (Regazzi \& Leite, 1992).

Através do presente trabalho, objetivou-se avaliar a influência da fonte, época e tipo de solo na determinação da condutividade elétrica, a partir da concentração iônica, utilizando-se a regressão linear e o teste de identidade de modelo.

\section{MATERIAL E MÉTODOS}

Os dados empregados na presente pesquisa, provêm do banco de dados gerado durante o programa Geração e Adaptação de Tecnologia (GAT), ocasião em que foram amostradas, mensalmente, águas de diferentes origens e épocas, em propriedades dos Estados do Ceará, Paraíba e Rio Grande do Norte, a partir do início de 1988 (Medeiros, 1992). O banco de dados se compõe de 562 análises oriundas de 55 propriedades rurais, sendo 17 no Rio Grande do Norte, 19 no Ceará e 19 na Paraíba. As determinações físico-químicas feitas nas amostras de águas, foram: $\mathrm{pH}, \mathrm{CE}, \mathrm{Ca}, \mathrm{Mg}, \mathrm{Na}, \mathrm{K}, \mathrm{Cl}, \mathrm{HCO}_{3}$, $\mathrm{CO}_{3} \mathrm{e} \mathrm{SO}_{4}$. No final de janeiro de 1988 a março de 1989 realizaramse amostragens nas propriedades, até 411 dias após o início das coletas.

Para este trabalho, determinaram-se 14 intervalos, de acordo com os dias de amostragem; para isto, calculou-se a amplitude total do tempo de amostragem em que a primeira amostragem foi feita aos 40 dias após o primeiro de janeiro de 1988 e a última 
aos 411 dias. A amplitude total foi de 371 e, determinada a raiz quadrada, obteve-se o número de intervalos (NI) de classe adequado para utilizar, caso em que o NI foi de 19,26, porém se dividiu o conjunto de dados em 14 intervalos, por se achar que o número de análises por intervalo seria ideal para o cálculo da regressão linear múltipla. Dividindo-se a amplitude total pelo número de intervalo, encontrou-se a amplitude do intervalo de classe, que foi de 26,5. Com base nesses dados, fez-se a distribuição das análises, de acordo com as épocas de amostragem (Tabela 1).

Tabela 1. Número de amostras de água analisadas por intervalo de tempo para 14 épocas estudadas

\begin{tabular}{ccc}
\hline Época & Intervalo de Classe (dia) & Número de Amostras \\
\hline E1 & $40,0-66,5$ & 34 \\
E2 & $66,5-93,0$ & 47 \\
E3 & $93,0-119,5$ & 36 \\
E4 & $119,5-146,0$ & 46 \\
E5 & $146,0-172,5$ & 40 \\
E6 & $172,5-199,0$ & 55 \\
E7 & $199,0-225,5$ & 48 \\
E8 & $225,5-252,0$ & 31 \\
E9 & $252,0-278,5$ & 33 \\
E10 & $278,5-305,0$ & 24 \\
E11 & $305,0-331,5$ & 48 \\
E12 & $331,5-358,0$ & 52 \\
E13 & $358,0-384,5$ & 47 \\
E14 & $384,5-411,0$ & 21 \\
\hline
\end{tabular}

\section{Análises estatísticas}

Ajustaram-se equações de regressão linear cuja variável dependente foi a condutividade elétrica (CE) e, independente, os íons analisados na água, de conformidade com a época de amostragem, fonte (tipo de manancial) e tipo de solo no local da amostragem. As equações ajustadas foram comparadas pelo teste de identidade de modelo para se verificar a hipótese de nulidade de que as equações são iguais estatisticamente. $\mathrm{O}$ teste de identidade de modelo baseia-se na diferença entre a soma dos quadrados de parâmetros do modelo completo e a soma de quadrados de parâmetros do modelo reduzido (Regazzi \& Leite, 1992). Inicialmente, fez-se o teste para todas as equações ajustadas para cada relação entre a $\mathrm{CE}$ e os íons por época, fonte (Tabela 2) e solo (Tabela 3); depois, o teste de identidade de modelo, comparando-se cada equação duas a duas, ou seja, aplicando-se o teste para cada época, fonte e solo, dois a dois. Neste trabalho a variável dependente foi a $\mathrm{CE}$, diferente de outros trabalhos da literatura, pois se chegou à conclusão de que, conceitualmente, a CE é que depende das concentrações dos íons na água porém, matematicamente, os

Tabela 2. Número de amostras analisadas por fonte de água e classe de solo local

\begin{tabular}{clc} 
& Fonte & Número de Amostras $^{(*)}$ \\
\hline F1 & Poço & 335 \\
F2 & Rio & 72 \\
F3 & Açude & 145 \\
\hline
\end{tabular}

\footnotetext{
(*) Foram desprezadas 10 análises de córrego
}

resultados podem ser comparados aos modelos descritos na literatura, que invertêm a posição para estimativa de concentrações iônicas. Para o teste de identidade de modelo utilizou-se o software SAEG, desenvolvido pela Universidade Federal de Viçosa.

Tabela 3. Número de análises por solo

\begin{tabular}{clc}
\hline & \multicolumn{1}{c}{ Solo } & Número de Amostras \\
\hline S1 & Neossolo Quartzarêmico & 10 \\
S2 & Luvissolo Crômico $^{(2)}$ & 199 \\
S3 & Cambissolo Háplico $_{\text {Seossolo Lítico }}$ & 33 \\
S4 & Neo & 84 \\
S5 & Latossolo Vermelho-Amarelo & 22 \\
S6 & Luvissolo Crômico ${ }^{(3)}$ & 75 \\
S7 & Planossolo Háplico & 20 \\
S8 & Neossolo Regolítico & 24 \\
S9 & Chernossolo Rêndzico & 17 \\
S10 & Planossolo Nátrico & 78 \\
\hline${ }^{2}$ Na classificacão antiga Bruno-não-Cálcico; ${ }^{3}$ Na classificação antiga Podzólico Vermelho- \\
Amarelo, EMBRAPA (1999).
\end{tabular}

\section{RESULTADOS E DISCUSSÃO}

\section{Avaliação por época de amostragem}

Utilizando-se o modelo de regressão linear simples para se avaliar a composição química da água de irrigação por época de amostragem, observou-se que, para $\mathrm{Ca}^{2+}$, os coeficientes de determinação variaram entre 0,269 - 0,785; para $\mathrm{Mg}^{2+}, \mathrm{K}^{+}$, $\mathrm{Na}^{+}, \mathrm{Cl}^{-}, \mathrm{HCO}_{3}^{-}, \mathrm{CO}_{3}{ }^{2-}$ e $\mathrm{SO}_{4}{ }^{2-}$; esses valores foram de 0,732 0,976, 0,028 - 0,843, 0,751 - 0,998, 0,677 - 0,994, 0,039-0,713, 0,058 - 0,763 e 0,170 - 0,966, respectivamente (Tabela 4). Verificase, também, que para algumas relações não houve significância para o coeficiente $b$ da equação linear para um coeficiente de determinação menor, o que se deve ao número de amostras em cada época de coleta.

Em épocas com maior número de dados, a média dos desvios diminui e a diferença pode ser significativa. Notadamente, observa-se existir variabilidade nos ajustes dos dados, em função da época de amostragem; inclusive, em algumas épocas para determinado íon, não houve significância na regressão ajustada, como é o caso de $\mathrm{CE}$ x $\mathrm{HCO}_{3}^{-}$em E11 e $\mathrm{CE} \times \mathrm{CO}_{3}{ }^{2-}$ em E10 e $\mathrm{CE} \times \mathrm{K}^{+}$em E5, cuja regressão ajustada também não foi significativa.

Alguns estudos chamam atenção para a necessidade de se observar a influência de tais fatores: Maia et al. (1998) e Medeiros (1992) atentam para que, ao se estimar algumas características químicas em função da condutividade elétrica, deve-se levar em consideração o local, a época de coleta da amostra e sua origem (manancial) pois, durante a época seca de algumas regiões, ocorre maior concentração de sais na água devido, em sua maior parte, às altas taxas de evaporação. Assim, em virtude dessa variabilidade de ajuste das regressões com a época de amostragem, ter-se-iam várias equações, devido a variação das concentrações dos íons com as condições climáticas, principalmente evaporação e precipitação e, como a CE também varia com as condições climáticas, a relação entre a mudança da CE e a concentração de determinado íon parece 
não ser linear, ou seja, uma diluição ou concentração dos íons na água causada na época das chuvas ou de alta evaporação não tenha, necessariamente, uma relação proporcional ao aumento ou diminuição da CE.

Teoricamente, a condutividade elétrica aumenta com a concentração de íons, porém se deve levar em consideração as constantes de solubilidade iônica $\left(\mathrm{K}_{\mathrm{ps}}\right)$ que, ao serem atingidas, os íons precipitam limitando a contribuição para o seu aumento; além disso, a CE não depende exclusivamente de um íon apenas, mas de todas as formas ionizadas presentes na água, como mostraram Maia et al. (2001) afirmando que a CE é multivariada e os melhores ajustes foram conseguidos utilizando-se a regressão linear múltipla porém, quando comparados os dados de regressão com os de outros autores (Medeiros, 1992; Martins et al., 1997) verifica-se que os valores de ajustes foram bem melhores que os obtidos por aqueles autores, com exceção para o cálcio, que apresentou ajuste inferior.

$\mathrm{Na}$ comparação das equações de regressão através da análise de identidade de modelo, foi feito primeiro para todas as regressões (Tabela 4) e, depois, comparando-se as equações duas a duas (Tabela 5) no total de 91 combinações para cada íon. Ao se comparar todas as equações ajustadas constatouse que, com exceção do sódio, os diversos íons apresentaram diferença estatística entre os modelos ajustados e, mesmo para o sódio, efeito de diluição no teste $\mathrm{F}$ na análise de identidade de modelo visto que, embora não sendo significativo quando comparados os 14 modelos constatou-se que, quando comparadas as épocas, duas a duas, $30 \%$ das comparações foram estatisticamente significativas. Para os demais íons, a diferença foi significativa entre algumas equações ajustadas por época, sendo as maiores diferenças verificadas para cloreto e cálcio com 46,15 e $40,66 \%$, respectivamente, enquanto as encontradas entre épocas foram para potássio, com apenas $15,38 \%$ das combinações possíveis que, por sua vez, foram diferentes estatisticamente pelo teste de identidade de modelo (Tabela 5).

Ajustados os dados dos íons analisados em função do tempo observa-se, nas Figuras 1 e 2 e na Tabela 6, que os dados de cálcio, magnésio, sódio, cloreto, bicarbonato e CE se ajustaram ao modelo cúbico, indicando a existência de um

Tabela 4. Parâmetros das equações de regressão linear, coeficientes de determinação e teste de identidade de modelos, por época

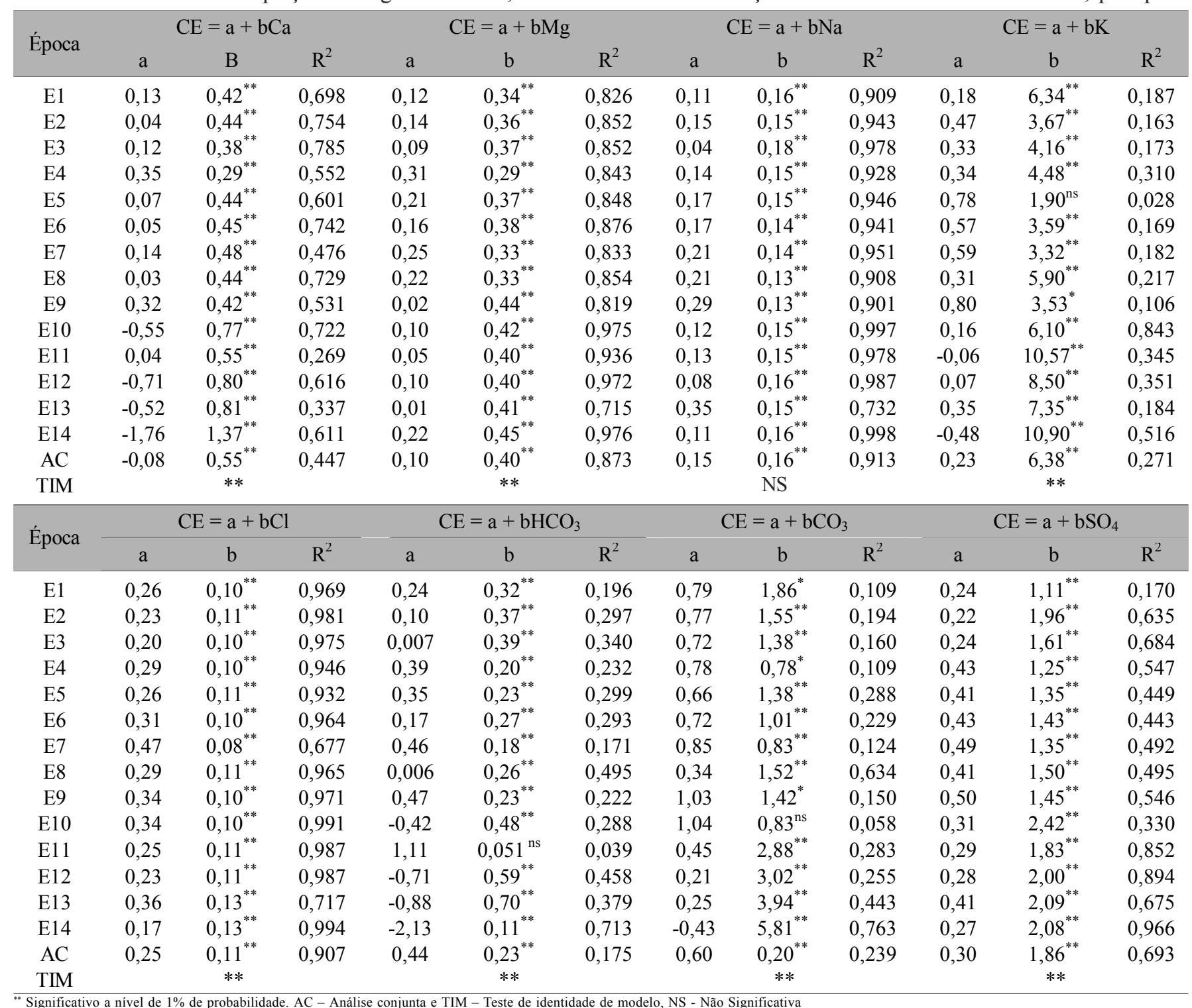


Tabela 5. Quantidade e percentagem de modelos comparados dois a dois estatisticamente, diferentes para as 14 épocas e íons analisados

\begin{tabular}{|c|c|c|c|}
\hline \multirow{2}{*}{ Íons } & \multicolumn{2}{|c|}{ Modelos } & \multirow{2}{*}{$\%$ Diferentes } \\
\hline & Iguais & Diferentes & \\
\hline $\mathrm{Ca}$ & 54 & 37 & 40,66 \\
\hline $\mathrm{Mg}$ & 64 & 27 & 29,67 \\
\hline $\mathrm{K}$ & 77 & 14 & 15,38 \\
\hline $\mathrm{Na}$ & 61 & 30 & 32,97 \\
\hline $\mathrm{Cl}$ & 49 & 42 & 46,15 \\
\hline $\mathrm{HCO}_{3}$ & 63 & 28 & 30,77 \\
\hline $\mathrm{CO}_{3}$ & 56 & 32 & 36,36 \\
\hline $\mathrm{SO}_{4}$ & 73 & 18 & 19,78 \\
\hline
\end{tabular}

Tabela 6. Equações de regressão para cálcio, magnésio, sódio, cloreto e bicarbonato e valores de $\mathrm{pH}$ e $\mathrm{CE}$ em função do tempo de amostragem (dia)

\begin{tabular}{lc}
\hline \multicolumn{1}{c}{ Equação } & $\mathrm{R}^{2}$ \\
\hline $\mathrm{Ca}=3,71-0,023^{* *} \mathrm{X}+0,000098^{* *} \mathrm{X}^{2}-0,00000012^{* *} \mathrm{X}^{3}$ & 0,89 \\
$\mathrm{Mg}=5,2-0,044^{* *} \mathrm{X}+0,0002^{* *} \mathrm{X}^{2}-0,00000024^{* *} \mathrm{X}^{3}$ & 0,86 \\
$\mathrm{Na}=9,86-0,067^{*} \mathrm{X}+0,00032^{*} \mathrm{X}^{2}-0,0000004^{*} \mathrm{X}^{3}$ & 0,83 \\
$\mathrm{Cl}=14,26-0,11^{* *} \mathrm{X}+0,00048^{* *} \mathrm{X}^{2}-0,00000056^{*} \mathrm{X}^{3}$ & 0,82 \\
$\mathrm{HCO}=4,02-0,025^{*} \mathrm{X}+0,00016^{* *} \mathrm{X}^{2}-0,00000025^{* *} \mathrm{X}^{3}$ & 0,74 \\
$\mathrm{pH}=7,44+0,0044^{* *} \mathrm{X}-0,0000082^{* *} \mathrm{X}^{2}$ & 0,78 \\
$\mathrm{CE}=1,68-0,0101^{* *} \mathrm{X}+0,000044^{* *} \mathrm{X}^{2}-0,000000048^{*} \mathrm{X}^{3}$ & 0,90 \\
\hline $\mathrm{Ca}, \mathrm{Mg}$ Na $\mathrm{Cl}, \mathrm{HCO}$ em mmol $/ \mathrm{L}$ e CE em dS/m**** Significativo a 5 e $1 \%$, respectivamente
\end{tabular}

ponto de máximo e outro de mínimo, ou seja, uma época de maior concentração e outra de menor concentração. Pelas condições climáticas que interferem na qualidade da água, o ajuste da equação cúbica mostrou que foi nas épocas de maior precipitação que se observaram as concentrações mais baixas, e nas épocas de maior evaporação, as maiores concentrações dos íons na água.

Pelos pontos de máxima e mínima das equações cúbicas e ponto de máximo para $\mathrm{pH}$ observa-se, na Tabela 7 , que as menores concentrações para cálcio, magnésio, sódio, cloreto e bicarbonato e valor de CE foram observadas, em média, aos 146 dias do dia primeiro de janeiro de 1988, sendo que esses
Tabela 7. Época após o início da amostragem de ocorrência de menor e maior concentração de cálcio, magnésio, sódio, cloreto e bicarbonato e valores de $\mathrm{pH}$ e $\mathrm{CE}$

\begin{tabular}{ccc}
\hline \multirow{2}{*}{ Íon } & \multicolumn{2}{c}{ Época (dia após inicio da amostragem) } \\
\cline { 2 - 3 } & Menor & Maior \\
\hline Cálcio & 171 & 373 \\
Magnésio & 151 & 404 \\
Sódio & 143 & 390 \\
Cloreto & 159 & 413 \\
Bicarbonato & 103 & 324 \\
pH & - & 268 \\
CE & 151 & 460 \\
\hline
\end{tabular}

valores variaram de 103 a 171 dias, indicando que as menores concentrações ocorreram em meados do mês de abril, com maior precipitação para as regiões estudadas; já as maiores concentrações iônicas foram observadas, em média, aos 394 dias após o inicio da coleta dos dados, variando de 324 a 460 dias. Neste caso, como as amostragens foram realizadas até os 411 dias após o dia 01/01/1988, o valor encontrado pela regressão está dentro do espaço amostral, porém aos 394 dias esses valores de maior concentração foram observados no mês de janeiro do ano seguinte ao início da amostragem. Para $\mathrm{CE}$, notou-se que o ponto de máximo ficou fora do espaço amostral, indicando que os maiores valores de CE tenderam a ser superiores no ano seguinte do início da amostragem.

É importante observar, na Figura 1, que os teores médios de bicarbonato foram superiores aos de cálcio e magnésio durante praticamente todo o período de amostragem, enquanto para na Figura 2 se observa que os teores médios de cloreto foram sempre superiores aos de sódio e esses foram sempre superiores aos teores médios de cálcio, magnésio e bicarbonato.

Os dados (Figuras 1 e 2 e Tabelas 6 e 7) representam a variabilidade temporal na concentração dos íons na água, corroborando com os dados apresentados nas Tabelas 4 e 5, em que pode o ajuste linear entre a $\mathrm{CE}$ e os íons variar também com a época da amostragem que está intimamente ligada às condições de precipitação e evaporação, o que não favorece quanto a precisão, ao se utilizar uma equação para estimação da concentração dos íons pela CE da água.

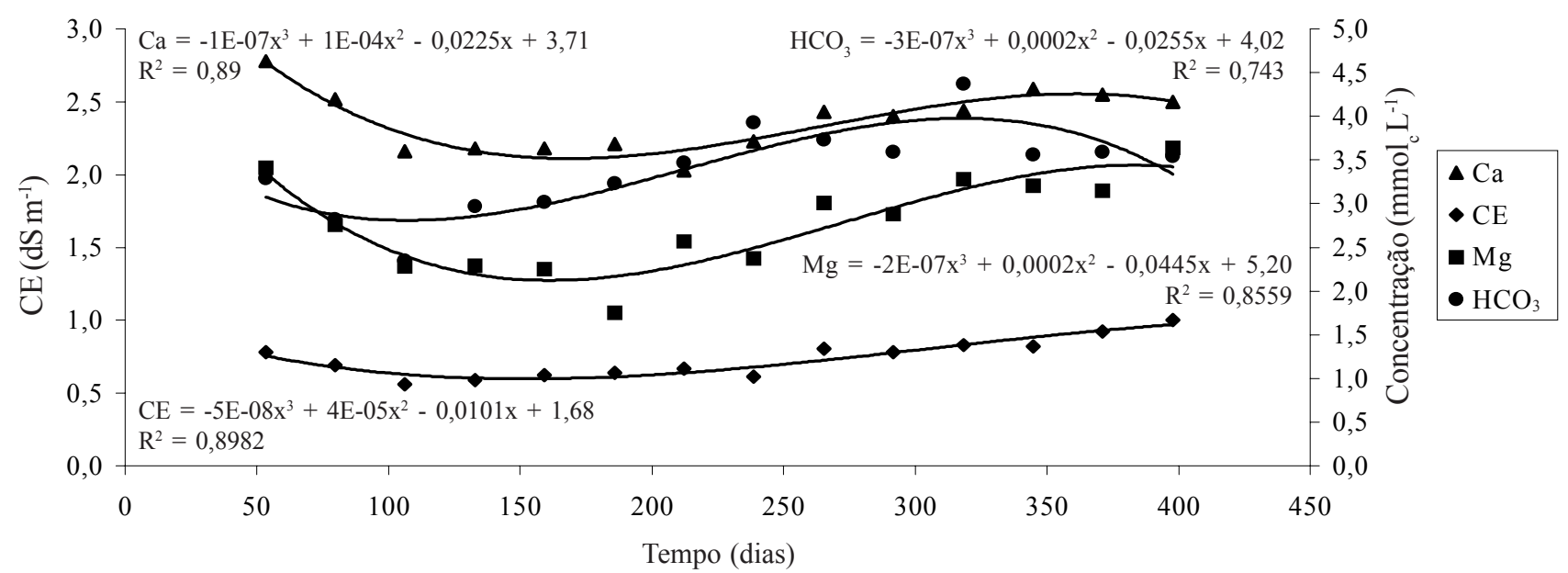

Figura 1. Concentração de cálcio, magnésio, bicarbonato e valores de condutividade elétrica $\left(\mathrm{dS} \mathrm{m}^{-1}\right)$ em função do tempo de amostragem 
A título de exemplo, admitam-se duas épocas distintas, E3 e E14; para determinar a concentração de cloreto para uma água com $\mathrm{CE}$ de 2,0 dS m-1 obtém-se, para E3 e E14, respectivamente, 18,0 e 14,08 $\mathrm{mmol}_{\mathrm{c}} \mathrm{L}^{-1}$, indicando que, para uma mesma $\mathrm{CE}$ a concentração de cloreto não é a mesma em todas as épocas estudadas. Nesta situação fica claro que para que os modelos estatísticos representem bem um fenômeno, é necessário se conhecer a verdadeira relação entre as variáveis dependente e independente, para que se possa fazer inferências estatísticas precisas com utilidade prática (Regazzi \& Leite, 1992).

\section{Avaliação por fonte}

$\mathrm{Na}$ Tabela 8 encontram-se os resultados das análises de regressão efetuadas com as águas das três fontes amostradas. Os dados indicam, para todos os íons, diferença estatística pelo teste de identidade de modelo e também, que a equação conjunta não representa os modelos ajustados para cada fonte, condição esta que indica que pelo menos dois modelos diferem estatisticamente, ou seja, pelo menos para duas fontes não se poderá utilizar a mesma equação para se estimar a CE pela concentração dos íons na água, neste caso e assim como para época, mesmo se agrupando as águas por fonte, os modelos diferem estatisticamente de fonte para fonte, concordando com a hipótese de que, para cada época e fonte, uma equação de regressão pode não explicar a variabilidade da concentração dos íons na água. Observa-se, então, que a fonte F1 (poço) apresentou menores coeficientes de determinação para todos os íons quando comparada com as demais fontes. Esta menor correlação para águas de poços deve ser atribuída ao fato de haver poços de diferentes aqüíferos com características geológicas distintas (Medeiros, 1992).

De forma análoga à época, as equações de regressão para fontes foram analisadas duas a duas, pelo teste de identidade de modelo e se verificou que, das 24 combinações possíveis para todos os íons analisados, 19 apresentaram diferenças significativas, indicando que, mesmo se ajustando os modelos para determinada fonte, esta pode diferir das outras fontes e,

Tabela 8. Parâmetros das equações de regressão linear ( $\mathrm{Y}=\mathrm{a}+\mathrm{bX}$ ), coeficientes de determinação e teste de identidade de modelos por fonte

\begin{tabular}{|c|c|c|c|c|c|c|c|c|c|c|c|c|}
\hline \multirow{2}{*}{ Fonte } & \multicolumn{3}{|c|}{$\mathrm{CE}=\mathrm{a}+\mathrm{bCa}$} & \multicolumn{3}{|c|}{$\mathrm{CE}=\mathrm{a}+\mathrm{bMg}$} & \multicolumn{3}{|c|}{$\mathrm{CE}=\mathrm{a}+\mathrm{bNa}$} & \multicolumn{3}{|c|}{$\mathrm{CE}=\mathrm{a}+\mathrm{bK}$} \\
\hline & $\mathrm{a}$ & $\mathrm{b}$ & $\mathrm{R}^{2}$ & $\mathrm{a}$ & $\mathrm{b}$ & $\mathrm{R}^{2}$ & $\mathrm{a}$ & $\mathrm{b}$ & $\mathrm{R}^{2}$ & $\mathrm{a}$ & B & $\mathrm{R}^{2}$ \\
\hline F1 & 0,25 & $0,39^{* *}$ & 0,540 & 0,28 & $0,35^{* *}$ & 0,665 & 0,20 & $0,14^{* *}$ & 0,728 & 0,59 & $5,02^{* *}$ & 0,118 \\
\hline $\mathrm{F} 2$ & $-0,18$ & $0,46^{* *}$ & 0,783 & 0,08 & $0,35^{* *}$ & 0,833 & 0,25 & $0,14^{* *}$ & 0,939 & 0,33 & $3,24^{* *}$ & 0,594 \\
\hline F3 & $-1,17$ & $1,24^{* *}$ & 0,649 & 0,02 & $0,42^{* *}$ & 0,977 & 0,11 & $0,15^{* *}$ & 0,995 & $-0,71$ & $10,89^{* *}$ & 0,488 \\
\hline Conjunta & $-0,082$ & $0,54^{* *}$ & 0,446 & 0,10 & $0,39^{* *}$ & 0,872 & 0,15 & $0,15^{* *}$ & 0,913 & 0,23 & $6,38^{* *}$ & 0,270 \\
\hline Identidade & \multicolumn{3}{|c|}{$* *$} & \multicolumn{3}{|c|}{$* *$} & \multicolumn{3}{|c|}{ * } & \multicolumn{3}{|c|}{$* *$} \\
\hline \multirow{2}{*}{ Fonte } & \multicolumn{3}{|c|}{$\mathrm{CE}=\mathrm{a}+\mathrm{bCl}$} & \multicolumn{3}{|c|}{$\mathrm{CE}=\mathrm{a}+\mathrm{bHCO}_{3}$} & \multicolumn{3}{|c|}{$\mathrm{CE}=\mathrm{a}+\mathrm{bCO}_{3}$} & \multicolumn{3}{|c|}{$\mathrm{CE}=\mathrm{a}+\mathrm{bSO}_{4}$} \\
\hline & a & $\mathrm{b}$ & $\mathrm{R}^{2}$ & $\mathrm{a}$ & $\mathrm{b}$ & $\mathrm{R}^{2}$ & a & $\mathrm{b}$ & $\mathrm{R}^{2}$ & a & $\mathrm{b}$ & $\mathrm{R}^{2}$ \\
\hline F1 & 0,40 & $0,10^{* *}$ & 0,736 & 0,94 & $0,08^{* *}$ & 0,057 & 0,86 & $0,10^{* *}$ & 0,145 & 0,57 & $1,30^{* *}$ & 0,439 \\
\hline $\mathrm{F} 2$ & 0,23 & $0,11^{* *}$ & 0,987 & $-0,74$ & $0,58^{* *}$ & 0,683 & 0,44 & $0,16^{* *}$ & 0,449 & 0,27 & $1,77^{* *}$ & 0,656 \\
\hline F3 & 0,12 & $0,12^{* *}$ & 0,987 & $-1,28$ & $0,97^{* *}$ & 0,726 & 0,26 & $0,58^{* *}$ & 0,601 & 0,30 & $2,11^{* *}$ & 0,901 \\
\hline Conjunta & 0,25 & $0,11^{* *}$ & 0,906 & 0,44 & $0,22^{* *}$ & 0,174 & 0,59 & $0,20^{* *}$ & 0,238 & 0,30 & $1,86^{* *}$ & 0,693 \\
\hline Identidade & & $* *$ & & & $* *$ & & & $* *$ & & & $* *$ & \\
\hline
\end{tabular}

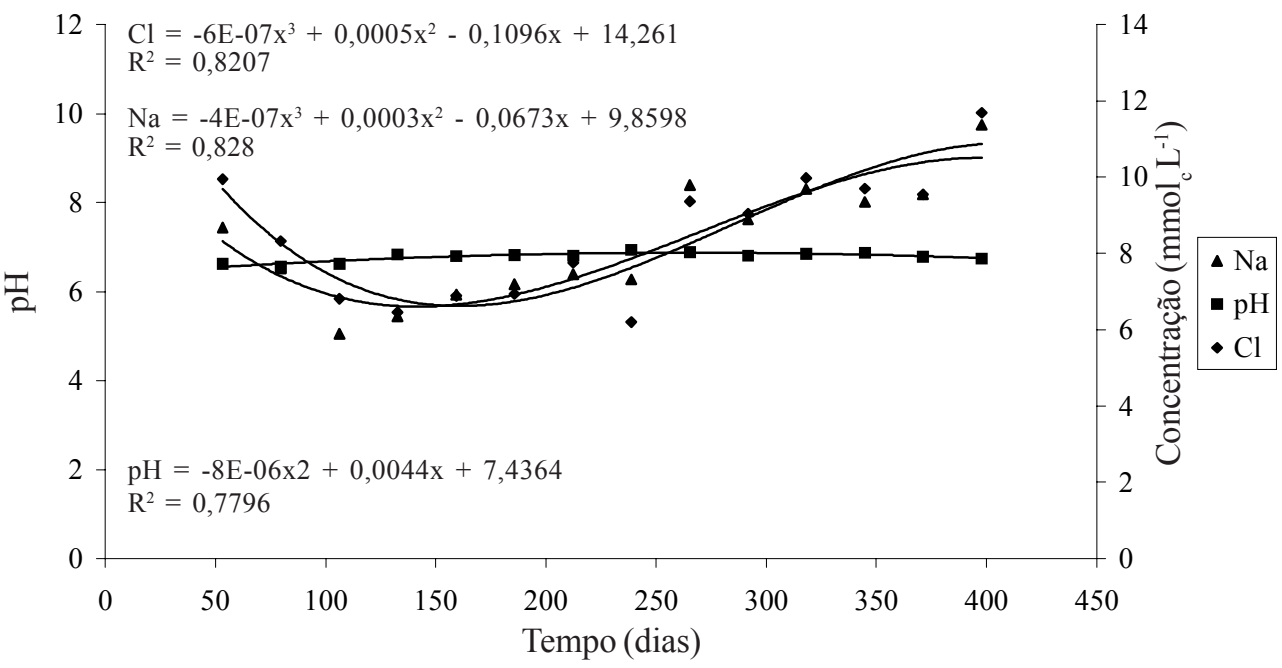

Figura 2. Concentração de sódio e cloreto e valores de pH, em função do tempo de amostragem. 
se for levada em consideração a variabilidade temporal, a probabilidade de adequação de um modelo ajustado para uma condição diminui a medida em que as equações diferem no espaço e no tempo. Leprun (1983) sugere utilizar-se equações diferentes para cada fonte e, para as condições do Nordeste do Brasil, em termos médios, as fontes variam da seguinte ordem: Açude $<$ Rio $<$ Poço Amazonas $<$ Poço Raso. Para exemplificar observa-se, na Tabela 9 que, para uma CE de 1,0 dS m-1, ocorre variação entre os teores ajustados para cada fonte, mostrando a necessidade de uma equação ajustada para cada fonte, caso esta explique a variabilidade dos dados, ou seja, apresente altos coeficientes de determinação, pois a capacidade preditiva de alguns modelos para potássio, bicarbonato e carbonato, principalmente, é baixa.

Tabela 9. Teores de sódio, cálcio, magnésio e cloreto obtidos pelos modelos de regressão ajustados para cada fonte para uma água com condutividade elétrica de $1,0 \mathrm{dS} \mathrm{m}^{-1}$

\begin{tabular}{|c|c|c|c|c|c|}
\hline \multirow{2}{*}{ Fonte* } & \multirow{2}{*}{$\mathrm{CE}\left(\mathrm{dS} . \mathrm{m}^{-1}\right)$} & \multicolumn{4}{|c|}{ Íon } \\
\hline & & $\mathrm{Na}^{+}$ & $\mathrm{Ca}^{+2}$ & $\mathrm{Mg}^{+2}$ & $\mathrm{Cl}^{-}$ \\
\hline $\mathrm{F} 1$ & 1,0 & 5,71 & 1,92 & 2,05 & 6,00 \\
\hline $\mathrm{F} 2$ & 1,0 & 5,35 & 2,41 & 2,62 & 6,41 \\
\hline F3 & 1,0 & 5,93 & 1,75 & 2,33 & 7,33 \\
\hline
\end{tabular}

\section{Avaliação por solo}

De acordo com os dados da Tabela 10, para cada íon analisado há uma variabilidade nos modelos ajustados, tanto nos valores dos interceptos como nos dos coeficientes angular e de determinação. Quando comparadas as equações pelo teste de identidade de modelo, observa-se que, com exceção do sódio, os demais íons apresentaram diferença estatística, ou seja, pelo menos dois modelos são diferentes estatisticamente, indicando a influência dos solos na composição iônica das águas.

Quando comparadas as equações duas a duas pelo teste de identidade de modelo, foi virtual a diferença estatística para um grande número de equações. (Tabela 11), na qual se observa, também, que mesmo não havendo diferença estatística para os solos no teste geral de identidade de modelo das equações de sódio, ao se comparar os modelos dois a dois, a diferença estatística aparece, indicando que no teste para os dez modelos, o sódio sofreu uma diluição do valor de $\mathrm{F}$, diminuindo seu valor e, portanto, não sendo significativo, situação semelhante que pode ocorrer e em que o teste $\mathrm{F}$ da análise de variância pode não mostrar significância verificando-se, quando se aplica um teste de contraste das médias, diferença significativa entre os tratamentos.

Tabela 11. Quantidade e porcentagem de modelos comparados dois a dois estatisticamente diferentes para os 10 solos estudados

\begin{tabular}{cccc}
\hline \multirow{2}{*}{ Íns } & \multicolumn{2}{c}{ Modelos } & \multirow{2}{*}{$\%$ Diferentes } \\
\cline { 2 - 3 } & Semelhantes & Diferentes & \\
\cline { 1 - 2 } $\mathrm{Ca}$ & 14 & 31 & 68,80 \\
$\mathrm{Mg}$ & 15 & 30 & 66,66 \\
$\mathrm{~K}$ & 17 & 28 & 62,22 \\
$\mathrm{Na}$ & 10 & 35 & 77,77 \\
$\mathrm{Cl}$ & 24 & 21 & 46,66 \\
$\mathrm{HCO}_{3}$ & 8 & 37 & 82,22 \\
$\mathrm{CO}_{3}$ & 14 & 31 & 68,80 \\
$\mathrm{SO}_{4}$ & 10 & 35 & 77,77 \\
\hline
\end{tabular}

Tabela 10. Parâmetros das equações de regressão linear, coeficientes de determinação e teste de identidade de modelos por solo

\begin{tabular}{|c|c|c|c|c|c|c|c|c|c|c|c|c|}
\hline \multirow{2}{*}{ Solo } & \multicolumn{3}{|c|}{$\mathrm{CE}=\mathrm{a}+\mathrm{bCa}$} & \multicolumn{3}{|c|}{$\mathrm{CE}=\mathrm{a}+\mathrm{bMg}$} & \multicolumn{3}{|c|}{$\mathrm{CE}=\mathrm{a}+\mathrm{bNa}$} & \multicolumn{3}{|c|}{$\mathrm{CE}=\mathrm{a}+\mathrm{bK}$} \\
\hline & $\mathrm{a}$ & $\mathrm{b}$ & $\mathrm{R}^{2}$ & $\mathrm{a}$ & $\mathrm{b}$ & $\mathrm{R}^{2}$ & $\mathrm{a}$ & $\mathrm{b}$ & $\mathrm{R}^{2}$ & $\mathrm{a}$ & $\mathrm{b}$ & $\mathrm{R}^{2}$ \\
\hline S1 & 0,18 & $0,13^{*}$ & 0,328 & 0,06 & $0,33^{\mathrm{ns}}$ & 0,141 & 0,11 & $0,18^{* *}$ & 0,534 & 0,21 & $0,65^{* *}$ & 0,669 \\
\hline S2 & $-0,04$ & $0,50^{* *}$ & 0,513 & 0,16 & $0,36^{* *}$ & 0,479 & 0,18 & $0,14^{* * *}$ & 0,546 & 0,48 & $2,99^{* *}$ & 0,046 \\
\hline S3 & 0,25 & $0,25^{* *}$ & 0,790 & 0,15 & $0,28_{* * *}^{* *}$ & 0,740 & 0,35 & $0,17^{* *}$ & 0,942 & 0,10 & $9,86_{* * *}^{* *}$ & 0,487 \\
\hline S4 & 0,39 & $0,26^{* *}$ & 0,341 & 0,35 & $0,31^{* *}$ & 0,739 & 0,14 & $0,14^{* *}$ & 0,913 & 0,57 & $2,69^{* *}$ & 0,193 \\
\hline S5 & $-0,06$ & $0,51^{* *}$ & 0,910 & 0,05 & $0,46^{* *}$ & 0,948 & $-0,19$ & $0,17^{\text {** }}$ & 0,975 & $-0,34$ & $18,41^{* *}$ & 0,671 \\
\hline S6 & 0,31 & $0,32^{* *}$ & 0,758 & 0,40 & $0,23^{* *}$ & 0,786 & 0,14 & $0,15^{* *}$ & 0,789 & 0,49 & $3,48^{* * *}$ & 0,154 \\
\hline S7 & 2,93 & $-0,14^{* *}$ & 0,078 & 2,77 & $-0,04^{\mathrm{ns}}$ & 0,010 & 1,25 & $0,07^{* *}$ & 0,863 & 0,34 & $-6,55^{* *}$ & 0,573 \\
\hline S8 & $-1,74$ & $1,75^{\mathrm{ns}}$ & 0,776 & 0,10 & $0,43^{* *}$ & 0,991 & 0,002 & $0,15^{* *}$ & 0,996 & $-1,54$ & $13,66^{* *}$ & 0,478 \\
\hline S9 & $-0,06$ & $0,42^{* *}$ & 0,699 & 0,22 & $0,29^{* *}$ & 0,863 & 0,30 & $0,14^{* *}$ & 0,934 & $-0,74$ & $15,96^{* *}$ & 0,640 \\
\hline $\mathrm{S} 10$ & $-0,09$ & $0,53^{* *}$ & 0,926 & 0,21 & $0,39^{* *}$ & 0,931 & 0,091 & $0,16^{* *}$ & 0,975 & 1,14 & $1,77^{* *}$ & 0,068 \\
\hline $\mathrm{AC}$ & $-0,08$ & $0,054^{* *}$ & 0,446 & 0,10 & $0,39^{* *}$ & 0,872 & 0,15 & $0,15^{* *}$ & 0,913 & 0,23 & $6,38^{* *}$ & 0,270 \\
\hline TIM & & $* *$ & & & $* *$ & & & ns & & & $* *$ & \\
\hline \multirow{2}{*}{ Solo } & \multicolumn{3}{|c|}{$\mathrm{CE}=\mathrm{a}+\mathrm{bCl}$} & \multicolumn{3}{|c|}{$\mathrm{CE}=\mathrm{a}+\mathrm{bHCO}_{3}$} & \multicolumn{3}{|c|}{$\mathrm{CE}=\mathrm{a}+\mathrm{bCO}_{3}$} & \multicolumn{3}{|c|}{$\mathrm{CE}=\mathrm{a}+\mathrm{bSO}_{4}$} \\
\hline & $\mathrm{a}$ & $\mathrm{b}$ & $\mathrm{R}^{2}$ & a & $\mathrm{b}$ & $\mathrm{R}^{2}$ & $\mathrm{a}$ & $\mathrm{b}$ & $\mathrm{R}^{2}$ & $\mathrm{a}$ & $\mathrm{b}$ & $\mathrm{R}^{2}$ \\
\hline $\mathrm{S} 1$ & 0,13 & $0,14^{* *}$ & 0,882 & 0,34 & $-0,01_{* *}^{\mathrm{ns}}$ & 0,001 & 0,33 & $-0,10^{\mathrm{ns}}$ & 0,036 & 0,25 & $1,15^{* *}$ & 0,103 \\
\hline S2 & 0,31 & $0,11^{* *}$ & 0,520 & 0,60 & $0,07^{* *}$ & 0,091 & 0,47 & $1,24^{* *}$ & 0,243 & 0,42 & $1,39^{* *}$ & 0,314 \\
\hline S3 & 0,41 & $0,10^{* *}$ & 0,933 & 0,61 & $0,19^{* *}$ & 0,173 & 1,34 & $0,03^{\text {ns }}$ & 0,212 & 0,55 & $0,89^{* *}$ & 0,454 \\
\hline S4 & 0,33 & $0,10^{* *}$ & 0,943 & 0,36 & $0,16^{* *}$ & 0,108 & 0,77 & $0,62^{* *}$ & 0,847 & 0,60 & $0,93^{* *}$ & 0,312 \\
\hline S5 & 0,22 & $0,10^{* *}$ & 0,991 & $-1,80$ & $1,30^{* *}$ & 0,753 & 1,44 & $0,68^{\mathrm{ns}}$ & 0,034 & 1,13 & $0,91^{\mathrm{ns}}$ & 0,059 \\
\hline S6 & 0,45 & $0,07^{* *}$ & 0,560 & $-0,18$ & $0,39^{* *}$ & 0,575 & 0,59 & $1,36^{* *}$ & 0,404 & 0,38 & $1,31^{* *}$ & 0,777 \\
\hline S7 & 0,34 & $0,10_{* *}^{* *}$ & 0,369 & 1,91 & $0,11_{* *}^{* *}$ & 0,680 & 2,11 & $0,55^{* *}$ & 0,539 & 1,82 & $0,64^{* *}$ & 0,773 \\
\hline S8 & 0,67 & $0,12^{* *}$ & 0,990 & $-2,59$ & $1,31^{* *}$ & 0,962 & 0,26 & $6,83^{* *}$ & 0,639 & 0,85 & $2,06^{* *}$ & 0,924 \\
\hline S9 & 0,28 & $0,10^{* *}$ & 0,970 & $-0,38$ & $0,51^{* *}$ & 0,476 & 0,67 & $1,42^{* *}$ & 0,171 & $-0,03$ & $1,97^{* *}$ & 0,823 \\
\hline $\mathrm{S} 10$ & 0,27 & $0,10^{* *}$ & 0,989 & 0,04 & $0,52^{* *}$ & 0,224 & 0,11 & $1,65^{* *}$ & 0,187 & 0,31 & $2,36^{* *}$ & 0,530 \\
\hline $\mathrm{AC}$ & 0,25 & $0,11^{* *}$ & 0,906 & 0,44 & $0,22^{* *}$ & 0,174 & 0,55 & $0,20^{* *}$ & 0,238 & 1,86 & $1,86^{* *}$ & 0,693 \\
\hline TIM & & $* *$ & & & $* *$ & & & ** & & & $* *$ & \\
\hline
\end{tabular}


Cruz \& Melo (1974) enumeram os principais fatores que contribuem para a salinização das águas subterrâneas do Nordeste, entre os quais se encontra a natureza geológica, ou seja, a influência litológica. Por outro lado, Leprun (1983) cita, como os principais indicadores de salinidade das águas superficiais, o tipo de solo e sua exposição à evaporação. Santos et al. (1984) afirmam que são os solos que mais decisivamente contribuem para a salinização das águas subterrâneas do freático, porém ressaltam que outros fatores e não somente a lixiviação dos sais solúveis dos solos, interferem na composição química das águas. Entre os fatores que contribuem para a composição iônica das águas pode-se citar a troca de bases, principalmente nos Luvissolo Crômico, Neossolo Regolítico, Planossolo Nátrico e Planossolo Háplico. Silva Júnior et al. (1999) correlacionaram algumas características das águas $\left(\mathrm{Na}^{+}\right.$, $\mathrm{Ca}^{2+}+\mathrm{Mg}^{2+}, \mathrm{Cl}^{-}$e RAS) em função da $\mathrm{CEa}$, para ao núcleos do sub-programa GAT nos Estados da Paraíba, Ceará e Rio Grande do Norte, tendo encontrado parâmetros de regressões lineares $\mathrm{Y}=\mathrm{a}+\mathrm{b}(\mathrm{CEa})$ com diferença significativa de $5 \%$ de probabilidade, para a mesma variável, entre núcleos e os dados de todas as águas estudadas. Os coeficientes de determinação $\mathrm{R}^{2}$ para a estimativa de alguns íons nas águas superficiais e subterrâneas foram, em geral, baixos, talvez pela maior variabilidade litológica. Para os núcleos estudados, os valores de $\mathrm{Cl}^{-}, \mathrm{Na}^{+}, \mathrm{Ca}^{2+}+\mathrm{Mg}^{2+}$ e RAS são mais confiavelmente estimados pela $\mathrm{CEa}$ quando se utilizam equações empíricas obtidas em cada núcleo, com exceção de teor de $\mathrm{Ca}^{2+}+\mathrm{Mg}^{2+}$ para o núcleo de Pau dos Ferros, RN.

\section{CONCLUSÕES}

1. Não existe precisão na estimativa da condutividade elétrica de águas de irrigação através de equações lineares ajustadas em função da concentração iônica de cálcio, magnésio, potássio, sódio, cloreto, bicarbonato, carbonato ou sulfato.

2. As equações lineares, ajustadas para a condutividade elétrica de águas em função de concentrações iônicas individuais, variaram com a época de amostragem da água, tipo da fonte e solo do local.

3. Os valores de condutividade elétrica e concentração dos íons cálcio, magnésio, sódio, cloreto e bicarbonato nas águas de irrigação, se ajustaram a um modelo cúbico de comportamento em função do tempo de amostragem.

\section{LITERATURA CITADA}

Ayers, R.S.; Westcot, D.W. A qualidade da água na agricultura. Campina Grande: UFPB, 1991.218p.

Costa, R.G.; Carvalho, H.O.; Gheyi, H.R. Qualidade da água de irrigação da microrregião homogênea de Catolé do Rocha,PB. Revista Brasileira de Ciência do Solo, Campinas, n.6, p.242244, 1982.

Cruciani, D.E. A drenagem na agricultura. São Paulo: Nobel, 1983.337p.

Cruz, W.B.; Melo, F.A.C.F. Estudos geoquímicos preliminares das águas subterrâneas do Nordeste do Brasil. Recife: SUDENE, 1974, 128p. Série hidrogeologia, 19
Farias, M.E. Comportamento ecofisiológico de Blutaparon portucolóides (St Hil) Mears, 1982 (Amaranthaceae) cultivada em diferentes concentrações da $\mathrm{NaCl}$. Porto Alegre: Universidade Federal do Rio Grande do Sul, 1985, 96p. Dissertação Mestrado

Leprun, J.C. Primeira avaliação das águas superficiais do Nordeste. Relatório de fim de convênio de manejo e conservação de solos do Nordeste brasileiro. Recife: SUDENE, 1983.

Maia, C.E.; Morais, E.R.C.de; Oliveira, M. de. Estimação de algumas características químicas da água de irrigação pela condutividade elétrica nas regiões da Chapada do Apodi e do Baixo Açu, Rio Grande do Norte. Caatinga, Mossoró; v.11, n.1/2, p.59-63, 1998.

Maia, C.E.; Morais, E.R.C. de Oliveira, M. de. Classificação da composição iônica da água de irrigação usando regressão linear múltipla. Revista Brasileira de Engenharia Agrícola e Ambiental, Campina Grande, v.5, n.1, p.55-59. 2001.

Martins, L.H. Avaliação da qualidade da água nos mananciais superficiais disponíveis para a irrigação na zona oeste potiguar. Mossoró: ESAM, 1993. 63p. Monografia Especialização

Medeiros, J.F. de. Qualidade da água de irrigação e evolução da salinidade nas propriedades assistidas pela "GAT" nos estados do RN, PB e CE. Campina Grande: UFPB, 1992. 173p. Dissertação Mestrado

Nunes Filho, J. El mejoramento de suelos sódicos y aguas bicarbonatadas, utilizando yeso agrícola en diferentes láminas de aplicación. Texcoco: Colégio de Postgraduados, 1984. 229p. Disertación Maestria.

Nunes Filho, J.; Sousa, A.R. de; Sá, V.A.L; Lima, B.P. Relações entre concentração de íons e a salinidade de águas subterrâneas e superficiais visando a irrigação, no Sertão de Pernambuco. Revista Brasileira de Engenharia Agrícola e Ambiental, Campina Grande. v. 4, n.2, p.189-193,2000.

Pizzarro, F. Drenaje agrícola y recuperación de suelos salinos. Madrid: Editorial Agrícola Española, 1978. 521p.

Regazzi, A.J.; Leite, H.G. Análise de regressão: teoria às aplicações em manejo florestal. Viçosa: UFV, 1992, 236p Mimeografado

Santos, J.P.; Azevedo, S.G.; Mistretta, G. Novos aspectos da salinização das águas subterrâneas do cristalino do Rio Grande do Norte. São Paulo: IPT, 1984. 27p.

Servant, M. J. Salinidad en los suelos y en las aguas sus carecterísticas y los problemas de irrigación y drenaje. Santo Domingo: El cañero, 1980. 16p. Boletim, 9

Silva Júnior, L.G.A.; Gheyi, H.R.; Medeiros, J.F. de. Composição química de águas do cristalino do Nordeste brasileiro. Revista Brasileira de Engenharia Agrícola e Ambiental, Campina Grande, v.3, n.1, p.11-17, 1999.

Thorne, J.; Rickeenback, R. El problema de las sales en el agua de riego, La Hacienda, Califórnia, n.6, p.7-12, 1972. 